\title{
VIEWPOINT
}

\section{Pacemaker syndrome: an iatrogenic condition}

\author{
Christopher M Travill, Richard Sutton
}

Pacemaker syndrome was first described in 1969 by Mitsui when it was referred to as the pacemaking syndrome. ${ }^{1}$ The name pacemaker syndrome was first coined by Erbel (using the German Schrittmacher syndrom) in $1979 .^{2}$ It can present with symptoms as severe as syncope, presyncope, oedema, dyspnoea, and chest pain, or more moderately and subtly as lethargy, palpitation, or an awareness of venous pulsation all of which may occur when there is atrial systole during ventricular systole. This is most frequent when there is ventriculoatrial conduction from the paced ventricle to the atrium usually via the atrioventricular node. ${ }^{34}$ The symptoms can be identical to those prompting implant. ${ }^{5}$ Occasionally pacemaker syndrome can occur in the absence of ventriculoatrial conduction when ventricular pacing is in competition with sinus rhythm. ${ }^{6}$

\section{Incidence of pacemaker syndrome}

The incidence of pacemaker syndrome varies with the vigour with which it is sought and it probably affects $7 \%$ of all ventricularly paced patients in its severe form in which it is essential to revise the pacemaker. If mild to moderate symptoms are considered it affects $20 \%$ of the ventricularly paced. This group too can benefit clinically from pacemaker upgrade. ${ }^{7}$ All too often the symptoms and signs of the syndrome are not sought and patients who previously experienced syncope, now relieved, complain little. This leads to a widespread impression that the syndrome is rare.

\section{Clinical diagnosis of pacemaker syndrome}

The diagnosis is made by reproduction of symptoms during ventricular pacing and depends on the history and the search for hypotension, signs of congestive cardiac failure, and venous cannon waves associated with ventricular pacing. Usually the diagnosis can be made clinically without recourse to special investigations. However, Doppler echocardiography can be useful in its diagnosis: Doppler ultrasound measurement of cardiac output in nine patients with ventriculoatrial conduction or symptoms consistent with pacemaker syndrome showed a $30 \%$ improvement when fully automatic (DDD) pacing rather than ventricular inhibited (VVI) pacing was used. In the remaining 20 patients with no history of pacemaker syndrome or ventriculoatrial conduction Doppler derived cardiac output improved by $14 \%$ when pacing was changed to DDD from VVI. ${ }^{8}$

\section{Haemodynamics of pacemaker syndrome}

Contraction of the atria against closed atrioventricular valves during ventricular systole leads to raised atrial pressures, loss of atrial contribution to ventricular filling, and a consequent fall in cardiac output which causes a fall in arterial pressure if the baroreceptor mediated rise in systemic vascular resistance is insufficient. ${ }^{9}$ In a study on 20 open-chest dogs with experimental complete heart block left atrial angiography showed retrograde blood flow into the pulmonary venous system at atrioventricular intervals of -50 and $-100 \mathrm{~ms}$. Therefore in addition to the loss of atrial contribution to ventricular filling there is a "negative atrial kick" further compromising haemodynamic function. ${ }^{10}$ In three patients studied by Alicandri et al ${ }^{11}$ there was an absent or smaller rise in peripheral resistance in response to a fall in cardiac output. The mechanism was thought to be due to vasodilatation in response to activation of atrial stretch receptors by atrial cannon waves which dominated over the baroreceptor mediated increase in resistance that occurs secondary to the fall in systemic arterial pressure. Similar findings were observed in a group of 20 patients studied haemodynamically more than 24 hours after coronary artery bypass grafting or aortic valve replacement. Hypotension with ventricular pacing occurred only in those patients with left atrial cannon waves. ${ }^{12}$ It has been suggested that patients with left ventricular disease, especially hypertrophy of any cause, are more sensitive to the correct timing of atrial systole and are, therefore more liable to be symptomatic with retrograde atrioventricular conduction. $^{\text {? }}$

A recent study of a large group of patients with intact ventriculoatrial conduction showed similar results, with peripheral resistance failing to rise in seven patients requiring upgrade to dual chamber mode because of symptoms of pacemaker syndrome whereas it rose during ventricular pacing in the symptom Westminster Hospital, Dean 2 AP.

Accepted for publication 12 February 1992 
free group. ${ }^{13}$ The difference between the response of the cardiac output between the two groups was negligible and the authors argued that the behaviour of the blood pressure was crucial. They advised that a cuff recording of blood pressure should be made at the onset of ventricular pacing during pacemaker implant and that a fall in systolic pressure of greater than $25 \mathrm{~mm} \mathrm{Hg}$ should be regarded as predictive for the possible development of pacemaker syndrome and they recommended dual chamber pacing. ${ }^{13}$ We suggest that if clinicians allow their choice of pacing mode to be guided by haemodynamic variables during temporary ventricular pacing before implant, they must assess the patient in the upright posture $\left(60^{\circ}\right.$ head up tilt with appropriate safety restraint) before considering it appropriate to implant a VVI unit. ${ }^{14}$

\section{Pacemaker syndrome in different pacing modes}

In a small group of patients, paced in VVI mode and presenting with symptoms of pacemaker syndrome, changing the mode from VVI to atrial inhibited (AAI) relieved symptoms and was associated with a fall in both the mean right atrial and pulmonary capillary wedge pressures as well as a rise in cardiac output. ${ }^{15}$ In view of the high prevalence of ventriculoatrial conduction in patients with sinus node disease, AAI pacing with the possible addition of a sensor-driven facility for those patients who do not show an increase in sinus rate on exertion (chronotropic incompetence) is advocated by some for all patients in whom atrioventricular conduction is sound. ${ }^{16}$

Two large randomised controlled trials to compare ventricular and dual chamber pacing in patients with sinus rhythm identified improved well-being even in "asymptomatic" patients when the dual chamber mode was used. ${ }^{17}{ }^{18}$ However, ventricular pacing is the preferred mode in patients with symptomatic bradycardia in the presence of atrial fibrillation.

Although the early dual chamber pacing mode (atrial synchronous ventricular inhibited (VDD)) was an improvement over the atrioventricular sequential (DVI) mode, it was still associated with pacemaker syndrome in those patients whose sinus rate dropped below the programmed lower rate at which point the pacing mode effectively became VVI ${ }^{19} \mathrm{~W}$ ith the advent of fully automatic dual chamber pacing systems (DDD) it became apparent that pacemaker syndrome can still occur in properly functioning dual chamber pacing systems in the DDD mode if the atrioventricular delay is programmed either too short or too long. ${ }^{20}$ The importance of the timing of left atrial depolarisation was addressed in a study of 16 patients with dual chamber pacemakers who had haemodynamic variables measured at different atrioventricular delays when they were supine and erect. ${ }^{21} \mathrm{~A}$ wide range of interatrial conduction delay $(70 \mathrm{~ms}-380 \mathrm{~ms}$ using oesophageal recording) between right atrial pacing artefact and left atrial depolarisation was seen and the optimal atrioventricular delay was more easily identified during $80^{\circ}$ head up tilt than with the patient supine. In those patients in whom the interatrial conduction delay exceeded $150 \mathrm{~ms}$ (three of 16 patients) a programmed atrioventricular delay of $150 \mathrm{~ms}$ resulted in left atrial activation after the ventricular spike, yielding left atrial contraction during left ventricular systole. The temporal difference between sensing of spontaneous right atrial activation and right atrial pacing is such that at a given atrioventricular interval, the sequence between atrial and ventricular contraction is longer in the atrial synchronous ventricular inhibited (VDD) than in the atrioventricular sequential mode (DVI). Some pacemakers now take this delay into account as a programmable feature. The DDI mode has been recommended for patients with carotid sinus syndrome, malignant vasovagal syndrome, and sick sinus syndrome who do not require atrial tracking (ventricular pacing as a result of atrial sensing) and who often have ventriculoatrial conduction. ${ }^{22-24}$ In this mode it is impossible for pacemaker mediated tachycardia to occur but pacemaker syndrome can occur with non-conducted premature atrial beats or with retrograde conduction from premature ventricular beats. ${ }^{25}$ Either of these events will inhibit atrial output if they occur outside the post ventricular atrial refractory period. They are then followed by a ventricular stimulus at the programmed ventricular rate which may be conducted retrogradely if the atrium has had sufficient time to recover. Ventricular pacing will only then be inhibited if the sinus rate recovers and is normally conducted. In patients with documented ventriculoatrial conduction the atrial refractory period in DDI should be programmed long enough to include the retrograde conduction interval. Thus sensing of a retrograde $P$ wave and atrial output inhibition is avoided. It is suggested that the atrial refractory period be set to $325 \mathrm{~ms}$ at programmed rates of 55-85 pulses per minute (ppm) shorter for faster rates, and longer for slower rates.

\section{Pacemaker syndrome in rate responsive} pacing

Sensor driven ventricular pacing (VVIR) would be expected to result in pacemaker syndrome at resting heart rates in the same way as non-sensor driven ventricular pacing. In patients with sinus node chronotropic incompetence, a group of patients who are often considered suitable for the VVIR mode, ventricular pacing is often programmed so as to be inhibited at rest but triggered by the sensor during exercise. This may result in pacemaker syndrome during exercise with retrograde atrial activation as the rate of the paced rhythm exceeds that of the intrinsic rhythm. ${ }^{26}$ Syncope on exercise has been reported even in the absence of regular ventriculoatrial conduction. ${ }^{27}$

Sensor driven AAI pacing causing pacemaker syndrome-The onset of pacemaker syndrome was reported during exercise in a patient with a 
dual atrioventricular nodal pathway in whom conduction through the fast anterograde pathway was prolonged to such an extent by concomitant therapy with disopyramide that conduction occurred over the slow pathway. ${ }^{28}$ This resulted in pacemaker syndrome during moderate exercise when the paced atrial event was conducted with a long spike $Q$ interval leading to atrial systole just after the preceding QRS during ventricular systole.

\section{Treatment of pacemaker syndrome}

Because symptoms of pacemaker syndrome overlap with those of pacemaker malfunction it is vital to exclude malfunction as the first step in investigation. Optimal therapy for symptomatic bradycardia of any cause is use of a pacing system that includes atrial sensing and pacing wherever atrial electrophysiology permits. In practice this will frequently be a dual chamber device where the atrial refractory period should be programmed to at least $25 \%$ longer than the measured ventriculoatrial conduction time to avoid pacemaker mediated or endless loop reentry tachycardia. Furman's group evaluated ventriculoatrial conduction in 432 patients receiving a permanent pacemaker. ${ }^{29}$ One hundred and sixty two had intact ventriculoatrial conduction including $14 \%$ of patients with complete anterograde atrioventricular block. Most patients with intact anterograde conduction had ventriculoatrial conduction. They evaluated these patients by means of incremental ventricular pacing and observed that the ventriculoatrial conduction interval was prolonged in most at faster rates with complete retrograde ventriculoatrial block at rates exceeding 120 pulses per minute in $50 \%$ of patients with intact atrioventricular conduction at rest. They estimated that programming the postventricular atrial refractory period to $300 \mathrm{~ms}$, an upper rate limit of $140 \mathrm{ppm}$ and an atrioventricular delay of $125 \mathrm{~ms}$ would prevent endless loop tachycardia in $90 \%$ of their patients.

Pacemaker syndrome can be avoided prospectively by choice of the most physiologically appropriate pacing mode for the individual patient. While this will inevitably lead to a higher incidence of dual chamber pacing and increased cost, the cost of upgrading as many as $20 \%$ of ventricularly paced patients must be considered together with the technical difficulties of passing a second lead and the increased risk of infection that is attendant upon second and subsequent pacemaker procedures. Increased cost need not be enormous if AAI pacing is considered as first choice mode for patients with sick sinus syndrome. Furthermore, the cost of many excellent dual chamber units has now fallen to within the range of many of the most sophisticated single chamber units. If improved physiological performance of dual chamber pacemakers with well demonstrated improved quality of life $\mathrm{e}^{1718}$ is ignored and patients most likely to have retrograde conduction are targeted the increased cost is moderate and comparable with the need for VVI upgrade. VVI pacing can still be considered for patients who are mostly in sinus rhythm and only show rare episodes of conduction defect and also for patients who are either severely disabled and show no retrograde conduction or who have a poor prognosis because of another disease.

As the use of dual chamber pacing systems with the ability to sense $P$ waves and at least one other physiological variable (by means of sensors such as those for respiratory minute volume and QT interval) increases, a far lower incidence of pacemaker syndrome should result. Furthermore, development of pacemaker artificial intelligence to diagnose the syndrome and automatic adjustment of the pacing mode to avoid it is expected within a few years.

\section{Alternatives to dual chamber pacing therapy of pacemaker syndrome}

(a) If pacemaker syndrome is encountered, it may be ameliorated by reducing the pacing rate so that competition between sinus rhythm and pacing is minimal with the possible addition of rate hysteresis in which pacing will only be triggered after a pause significantly longer than the pacing interval. ${ }^{30}$

(b) Antiarrhythmic drugs may be used to block retrograde atrioventricular conduction. Flecainide has previously been used for this purpose but in the light of the CAST study we do not think that it can be recommended. ${ }^{31} \mathrm{It}$ and other antiarrhythmic drugs can also paradoxically result in artificial circus movement tachycardias by prolonging the retrograde atrio-ventricular conduction time without complete retrograde block and may result in a retrograde $P$ wave occurring outside the atrial refractory period thereby precipitating pacemaker mediated tachycardia. ${ }^{32}$

(c) In those patients who are not pacemaker dependent and in whom the pre-implant symptoms are minor serious consideration should be given to the device being turned off and subsequently, if appropriate, explanted.

\section{New approach to the identification of potential pacemaker syndrome}

If a policy of DDD pacing for all suitable patients is not adopted a reliable test to identify patients at risk of developing pacemaker syndrome would be clinically valuable.

Atrial natriuretic peptide was discovered in 1981 and is released directly into the circulation in response to atrial wall stretch within the heart and it has potent vasodilator properties in addition to natriuretic and diuretic ones. ${ }^{33}$ Peripheral venous plasma atrial natriuretic peptide concentrations were measured in patients with DDD and VVI pacemakers and they were significantly lower in both the short and long term when the DDD mode was in operation and the concentrations were similar to those observed in controls. ${ }^{34}$ This difference is maintained during exercise. ${ }^{35}$ Concentrations of atrial natriuretic peptide were lower at physiological delays than at either long or short atrioventricular delays. ${ }^{36}$ Atrial natriuretic 
peptide concentrations were measured in patients with ventriculoatrial conduction both with and without symptoms of pacemaker syndrome. Concentrations were normal during sinus rhythm or DDD pacing (atrioventricular delay $150 \mathrm{~ms}$ ) in patients with pacemaker syndrome but rose to 7-8 fold during ventricular pacing with intact ventriculoatrial conduction. ${ }^{37}$ These data prompt reconsideration of the mechanism of hypotension in pacemaker syndrome. Animal studies ${ }^{38}$ (which have not been reproduced by others ${ }^{39}$ ) offer only weak support to the suggestions of Alicandri et al, ${ }^{11}$ Erlebacher et $a l,,^{12}$ and Witte $e t a l,{ }^{13}$ that the mechanism is an exaggerated or inappropriate neural reflex. Atrial natriuretic peptide has well defined vasodilator properties at concentrations seen in patients with symptomatic pacemaker syndrome. These effects may be additive to the neural reflex or may be the sole mechanism of inappropriate vasodilation. The higher concentrations found in patients with symptomatic pacemaker syndrome compared with those who had retrograde atrioventricular conduction without symptoms supports the involvement of an endocrine mechanism in this syndrome.

Pacemaker syndrome particularly in its milder form probably has a higher incidence and prevalence than is currently realised. It may be avoided by carefully programmed dual chamber pacing for all patients with normal atrial activity and atrioventricular conduction disease and by atrial pacing in patients with intact atrioventricular conduction. Pacemaker upgrade should be avoided on grounds of patient discomfort (including infection risk), operator difficulty in passing a second lead, and cost. Thus a policy of VVI pacing for all is no longer tenable.

1 Mitsui T, Hori M, Suma K, Wanibuchi Y, Saigusa M. The "pacemaking syndrome" [abstract]. In: Jacobs JE, eds. Proceedings of the eighth annual international conference on medical and biological engineering. Chicago: Association for the Advancement of Medical Instrumentation. 1969;29:3.

2 Erbel R. Pacemaker syndrome. Am J Cardiol 1979;44:771-2.

Werres R, Parsonnet V, Gilbert L, Zucker IR. Symptomatic unilateral cannon "a" waves in a patient with a ventricula unilateral cannon "a" waves in a pa

4 Johnson AD, Kaiken SL, Engler RL. Hemodynamic compromise associated with ventriculo-atrial conduction folpromise associated with ventriculo-atrial conduction following transven

5 Amikam S, Riss E. Untoward haemodynamic consequences of permanent ventricular pacing associated with ven triculo-atrial conduction. In: Meere C, ed. Proceedings of VI world symposium on cardiac pacing. PACESYMP 1979, chapter 15-6.

6 Nishimura RA, Gersh BJ, Vlietstra RE, Osborn MJ, Ilstrup DM, Holmes DR. Haemodynamic and symptomatić consequences of ventricular pacing. $P A C E$ 1982;5:903-10.

7 Ausubel K, Furman S. The pacemaker syndrome. Ann Intern Med 1985;103:420-9.

8 Stewart WJ, Dicola VC, Harthorne JW, Gillam LD, Weyman AE. Doppler ultrasound measurement of cardiac output in patients with physiologic pacemakers. $\mathrm{Am}$ Cardiol 1984;54:308-12.

9 Nishimura RA, Gersh BJ. Holmes DR Jr, Vlietstra RE, Broadbent JC. Outcome of dual-chamber pacing for the pacemaker syndrome. Mayo Clin Proc 1983;58:452-6.

10 Naito M, Dreifus LS, David D, Michelson EL, Mardelli TJ, Kmetzo JJ. Reevaluation of the role of atrial systole to cardiac haemodynamics: Evidence for pulmonary venous regurgitation during abnormal atrioventricular sequencing. Am Heart J 1983;105:295-302.

11 Alicandri C, Fouad FM, Tarazi RC, Castle L, Morant V. Three cases of hypotension and syncope with ventricular pacing: possible role of atrial reflexes. Am J Cardiol 1978; 42:137-42.

12 Erlebacher JA, Danner RL, Stelzer PE. Hypotension with ventricular pacing: an atrial vasodepressor reflex in human ventricular pacing: an atrial vasodepresso

13 Witte J, Bondke H, Muller S. The pacemaker syndrome: a haemodynamic complication of ventricular pacing. Cor Vasa 1988;30:393-9.

14 Fitzpatrick AP, Travill CM, Vardas PE, et al. Recurrent symptoms after ventricular pacing in unexplained syncope. PACE 1990;13:619-24.

15 El Gamal MIH, Van Gelder LM. Chronic ventricular pacing with ventriculo-atrial conduction versus atrial pacing in three patients with symptomatic sinus bradycardia. PACE 1981;4:100-5.

16 Ryden L. Atrial inhibited pacing - an underused mode of cardiac stimulation. PACE 1988;11:1375-9.

17 Kruse I, Arnman K, Conradson TB, Ryden LA. A comparison of the acute and long-term haemodynamic effects of ventricular inhibited and atrial synchronous ventricular inhibited pacing. Circulation 1982;65:846-55.

18 Perrins EJ, Morley CA, Chan SL, Sutton R. Randomised controlled trial of physiological and ventricular pacing. $\mathrm{Br}$ Heart $J 1983 ; 50: 112-7$.

19 Levine PA, Seltzer JP, Pirzada FA. The "pacemaker syndrome" in a properly functioning physiological pacing drome" in a properly functioni
system. $P A C E$ 1983;6:279-82

20 Torresani J, Ebagosti A, Allard-Latour G. Pacemaker syndrome with DDD pacing. PACE 1984;7:1148-51.

21 Wish M, Fletcher RD, Gottdiener JS, Cohen AI. Import ance of left atrial timing in the programming of dua chamber pacemakers. Am J Cardiol 1987;60:566-71.

22 Floro J, Castellanet M, Florio J, Messenger J. DDI: A new mode for cardiac pacing. Clin Prog Pacing Electrophysiol 1984;2:255-60.

23 Barold SS. The DDI mode of cardiac pacing. PACE 1987; 10:480-4.

24 Sutton R. Pacing in patients with carotid sinus and vasovagal syndromes. PACE 1989;12:1260-3.

25 Cunningham TM. Pacemaker syndrome due to retrograde conduction in a DDI pacemaker. Am Heart J 1988;115: 478-9.

26 Liebert HP, O'Donoghue S, Tullner WF, Platia EV. Pacemaker syndrome in activity-responsive VVI pacing. Am J Cardiol 1989;64:124-6.

27 Wish M, Cohen A, Swartz J, Fletcher R. Pacemaker syndrome due to a rate-responsive ventricular pacemaker. syndrome due to a rate-respon
$J$ Electrophysiol 1988;2:504-7.

28 Den Dulk K, Lindemans FW, Brugada P, Smeets JLRM, Wellens HJJ. Pacemaker syndrome with AAI rate variable pacing: importance of atrioventricular conduction properties, medication and

29 Klementowicz P, Ausubel K, Furman S. The dynamic nature of ventriculo-atrial conduction. $P A C E$ 1986;9: 1050-54.

30 Preston TA, Preston AW Jr. The automatic rate adjustment pacemaker. The possibilities of rate hysteresis. PACE 1978;1:178-85.

31 The CAST investigators, Preliminary report: Effect of encainide and flecainide on mortality in a randomized trial of arrhythmia suppression and myocardial infarction. $N$ Engl J Med 1989;321:406-12.

32 Bathen J, Gundersen T, Forfang K. Tachycardias related to atrial synchronous ventricular pacing. $P A C E$ 1982;5: 471-5.

33 De Bold AJ, Borenstein HB, Veress AT, Sonnenberg H. A rapid and potent natriuretic response to intravenous injection of atrial myocardial extract in rats. Life Sci 1981;28:89-94.

34 Vardas PE, Travill CM, Williams TDM, Ingram $A$, Lightman SL, Sutton R. Effect of dual chamber pacing on Lightman SL, Sutton R. Effect of dual chamber pacing on raised plasma atrial natriuretic peptide concentratio
complete atrioventricular block. $B M J 1988 ; 296: 94$.

35 Stangl K, Weil J, Seitz K, Laule M, Gerzer R. Influence of AV synchrony on the plasma levels of atrial natriuretic peptide (ANP) in patients with total AV block. PACE 1988;11:1176-81.

36 Noll B, Krappe J, Goke B, Maisch B. Influence of pacing mode and rate on peripheral levels of atrial natriuretic peptide (ANP). PACE 1989;12:1763-68.

37 Travill CM, Williams TDM, Vardas $P$, et al. Hypotension in pacemaker syndrome is associated with marked atrial natriuretic peptide (ANP) release [abstract]. PACE 1989;12:93.

38 Edis AJ, Donald DE, Shepherd JT. Cardiovascular reflexes from stretch of pulmonary vein-atrial junctions in the dog. Circ Res 1970;27:1091-100.

39 Carswell F, Hainsworth R, Ledsome JR. Effects of distension of the pulmonary vein-atrial junctions upon peripheral vascular resistance. $J$ Physiol 1970;207:1-14. 